\title{
Time reduction in implicit single point incremental sheet forming simulation by refinement - derefinement
}

\author{
A. Hadoush ${ }^{1}$, A.H. van den Boogaard ${ }^{2}$ \\ ${ }^{1}$ Netherlands Institute for Metals Research, Mekelweg 2, P.O. Box 5008, 2600 GA Delft, The Netherlands \\ URL: $\underline{\text { www.nimr.nl }}$ \\ e-mail: a.hadoush@nimr.nl \\ ${ }^{2}$ Faculty of Engineering Technology, University of Twente, P.O. Box 217, 7500 AE Enschede, \\ The Netherlands \\ URL: $w w w$. utwente.nl \\ e-mail: a.h.vandenboogaard@ctw.utwente.nl
}

\begin{abstract}
This paper presents the implementation of a refinement - derefinement (RD) approach to reduce the computing time in single point incremental sheet forming (SPIF) simulation. The results of this approach are compared to a reference model that has a fine enough mesh to satisfy the process requirements. The fine mesh is created by refining an intermediate coarse mesh entirely. The RD approach performs one level of refinement-derefinement on the same intermediate coarse mesh based on a geometrical error indicator. The integration point data is mapped by a least squares method. The simulation of forming a 45 degree pyramid is considered as reference test. The refinement-derefinement approach reduces the computing time for the reference model by $50 \%$. The achieved equivalent plastic strain by this approach shows a good agreement with the reference model.
\end{abstract}

Key words: Incremental Forming, Refinement - Derefinement.

\section{INTRODUCTION}

Nowadays, FE simulation of metal forming has been used by industry to better predict the geometry of their products and their structural behaviour. Because single point incremental forming (SPIF) is a dieless process, it is perfectly suited for prototyping and small volume production. SPIF is a displacement controlled process performed on a CNC machine. A clamped blank is deformed by the movement of the tool that follows a certain tool path, a sketch for SPIF is shown in Figure 1.

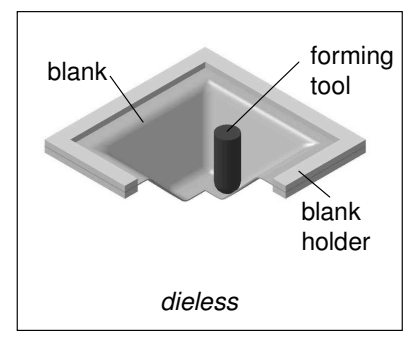

Fig. 1. SPIF process [1]

The SPIF numerical simulation faces several challenges e.g. the simulation computing time, the material model as well as the large deformation achieved by the process. The implicit simulation of SPIF provides a very good agreement with experimental data [1]. This paper presents a refinement-derefinement (RD) strategy to get over the necessity of having an initially fine mesh, would result in an enormous computing time. A fine mesh in the vicinity of the forming tool is required since there is a small contact area between the forming tool and the blank compared to the blank area. This fact hints that part of the blank needs to have a fine mesh while the rest of the blank can have a coarse mesh. During the process, the mesh connectivity is continuously changing, because of the moving tool. The main goal of the approach is to keep the number of elements as low as possible during the entire simulation.

\section{REFINEMENT - DEREFINEMENT}

h-adaptivity is used to perform refinement and derefinement [2]. The strategy consists of adapting the number of grid points and changing the connectivity. Grid points are added to areas where 
more accuracy is demanded and can be deleted in areas where the solution is accurate enough. In this paper, the nominated elements for refinement and its neighbours are refined once and will be called mother elements. Considering linear triangular elements, each mother element is divided into 4 elements, the new elements will be called refined elements. To preserve mesh compatibility, the neighbour elements of the mother elements are split into 2 elements. Any 2 split elements born out of the same mother element can be united and refined into refined elements [3]. Meanwhile, the derefinement performs only on the refined and split elements. It degenerates the previous connectivity in a reverse order. An example for element generation and degeneration is shown in figure 2 .
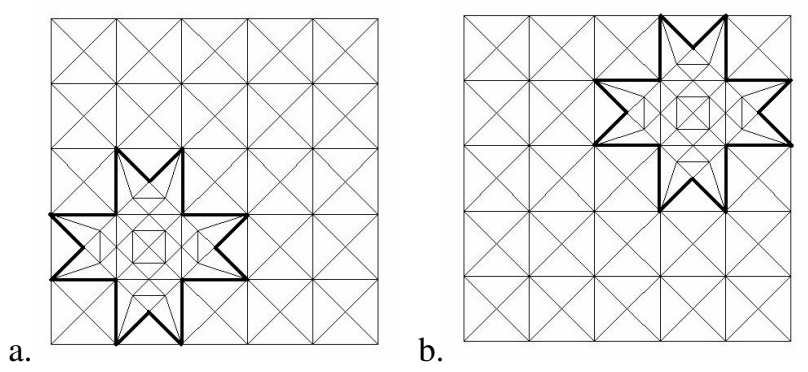

Fig. 2. Mesh generation a) refinement takes place at the lower left part, b) the lower left part is derefined and the upper right is refined

\subsection{Remeshing criterion}

An error indicator is used instead of an error estimator since it is computationally cheaper. Particularly, the geometrical error indicator is used. It measures the variation of the geometry within the blank [4]. Briefly, a set of tangent axes is determined for each element. The variation of these sets of tangents from one element to its neighbouring elements indicates the variation of the geometry. A nodal averaging technique is used to quantify this variation. If the variation within a group of elements exceeds a certain user input value, that group of elements is refined. Opposite wise, if the variation within a group of refined elements is less than the user input value, the derefinement takes place on that group.

\subsection{Data mapping}

When a new grid connectivity is created, the state variables have to transfer from the old mesh to the new mesh. There are several approaches. One approach is using the old nodal value to evaluate the new nodal value, then determine the state variables at the integration points [3]. Another approach is to use a patch recovery, that depends on selecting specific locations within a group of elements, creating a smoothed field out of it and evaluate the new data [5]. The chosen approach is the least squares method. The method fits a linear field based on all available integration points. The location of the integration points and its values are used to create the linear field, then the location of the new integration point is used to determine its value. Regardless the approach, data transfer predicts the exact value for the state variables when it maps mother element into split element or refined element. However it either over estimates or under estimates the new value for the following derefined cases: split into refined, refined into split, refined into mother and split into mother. The error is introduced because of fitting piecewise linear fields into one linear field. The least squares method is the optimum approach. It minimizes the error during data transfer and it is not computationally expensive compared to the other approaches.

\subsection{RD implementation test}

The RD approach is coded in the in-house implicit FE package DiekA. The correctness of the implementation has to be checked before going for SPIF simulation. For that purpose, an initial linear field for the equivalent plastic strain is prescribed for a FE strip model. The strip experiences 648 RD combination. Theoretically and numerically within the machine accuracy, the linear field must remain the same. This is achieved in the RD implementation test as shown in figure 3 .

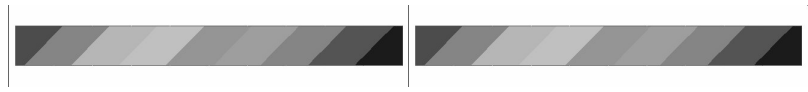

Fig. 3. The initial linear field of the equivalent plastic strain (left) and the final field (right).

\section{CASE STUDY}

To verify whether the RD approach leads to CPU time reduction, a $45^{\circ}$ pyramidal shape simulation is chosen as case study. An analytical spherical tool of $10 \mathrm{~mm}$ radius is used. The tool follows a counter clockwise tool path for 34 loops, it moves $0.5 \mathrm{~mm}$ vertically downward for each loop. The pyramidal shape is made out of a $100 * 100 * 1.2 \mathrm{~mm}$ initially 
flat blank. The blank is fully clamped at the edges.

\subsection{Discretization}

An intermediate coarse mesh is created, that consists of 800 linear triangular elements. The mesh is entirely refined producing 3200 elements. The latest mesh will be called the reference mesh. The reference mesh is used to perform the reference simulation. The intermediate coarse mesh is used in the RD approach and will be called the RD mesh. The RD approach controls the generation or the degeneration of elements from the RD mesh to reference mesh.

Two element types are used: discrete Kirchhoff triangle DKT and discrete shear triangle DST $[6,7]$. Both elements have 6 degrees of freedom per node. The elements contain both bending and membrane stiffness. It has 3 integration points in plane and 5 in thickness. DST is implicitly coupled by transverse shear stiffness of the material, while DKT is explicitly coupled by enforcing zero transverse shear strain at selected locations [5].

\subsection{Material model}

In order to focus on the numerical technique, the material model is kept as simple as possible. The isotropic yield behaviour of the material is modelled by the von Mises criterion. The elastoplastic nonlinear hardening is governed by the Nadai relation as follows in Equation 1

$$
\sigma=C\left(\varepsilon+\varepsilon_{0}\right)^{n}=500(\varepsilon+0.00243)^{0.2}
$$

Where $\sigma$ and $\varepsilon$ are the flow stress and the plastic strain respectively. The material has Young's modulus of $200 \mathrm{GPa}$ and Poisson's ratio of 0.3 . For realistic calculation, the authors acknowledge that a better material model is required, that includes the anisotropic behaviour of the sheets and the cyclic mode of deformation.

\section{RESULTS AND DISCUSSION}

At first, there is no significant difference in the achieved equivalent plastic strain by DKT or DST elements. DKT requires $40 \%$ less computing time than DST for the same simulation. The equivalent plastic strain for the middle integration point in thickness direction is plotted in figure 4. The achieved maximum strain in the reference simulation is 0.438 ; the RD simulation over estimates it by $6 \%$. A comparable strain distribution is achieved, but less smooth. As expected, a similar situation is observed for the plastic strain component $x x$ as plotted in figure 5 .
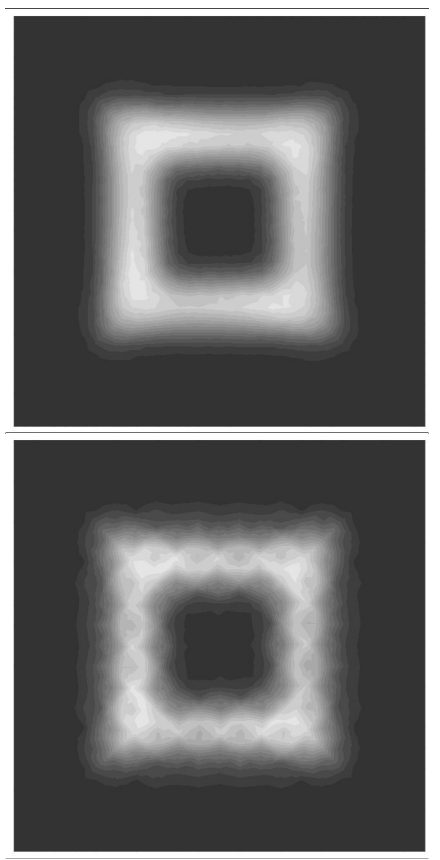

Fig. 4. DKT element, the equivalent plastic strain at the mid plane for the reference (top) and the RD (bottom)



Fig. 5. DKT element, the plastic strain component $x x$ at the mid - plane for the reference (top) and the RD (bottom)

However, at the outer integration points, the RD approach yields less accurate results . The maximum equivalent plastic strain is 0.837 in the reference simulation, the RD simulation over estimates it by $21 \%$ as plotted in figure 6 . The reason for that is the 
equivalent plastic strain and the flow stress are mapped independently and regardless the nonlinearity that relate the stress to the strain. The frequent smoothing of the flow stress results in over estimation of the plastic strain for the following steps in an incremental way.

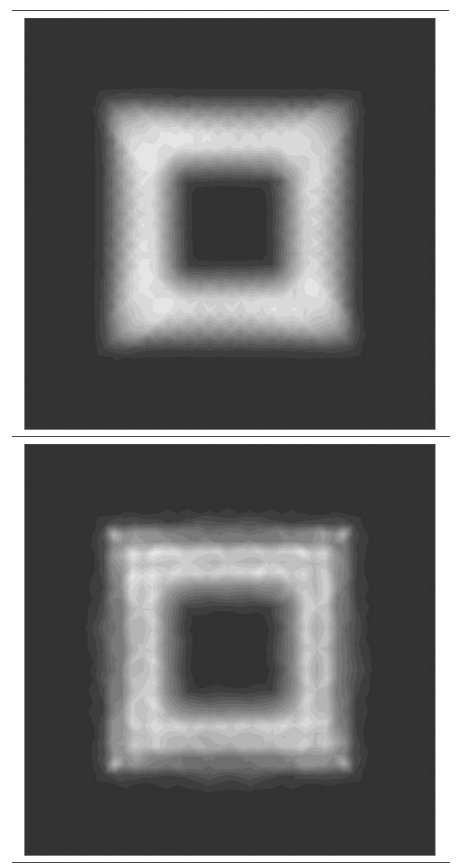

Fig. 6. DST element, the equivalent plastic strain at the highest integration point for the reference (top) and the RD (bottom)

The performance of the simulations is listed in table 1 and 2 for DKT and DST respectively. The RD performs well. It achieves almost 50\% reduction in computing time compared to the reference simulation for both elements. As plotted in figure 7, the reduction in computing time is achieved by keeping the number of nodes and consequently the number of elements as low as possible. The RD mesh is fine enough for the first couple of loops. Then, the geometrical variation within the blank triggers overall mesh growth, in order to capture the present curvature of the blank in the region of the tool path.

Table 1. Simulation performance for DKT element.

\begin{tabular}{|l|l|l|}
\hline & Reference & RD \\
\hline No. of steps & 8087 & 8154 \\
\hline No. of elements & 3200 & $800-2014$ \\
\hline No. of nodes & 1681 & $441-1048$ \\
\hline Computing time & $19.9 \mathrm{hr}$ & $9.93 \mathrm{hr}$ \\
\hline
\end{tabular}

Table 2. Simulation performance for DST element.

\begin{tabular}{|l|l|l|}
\hline & Reference & RD \\
\hline No. of steps & 8088 & 8168 \\
\hline No. of elements & 3200 & $800-2014$ \\
\hline No. of nodes & 1681 & $441-1048$ \\
\hline
\end{tabular}

\begin{tabular}{|l|l|l|}
\hline Computing time & $32.3 \mathrm{hr}$ & $15.8 \mathrm{hr}$ \\
\hline
\end{tabular}

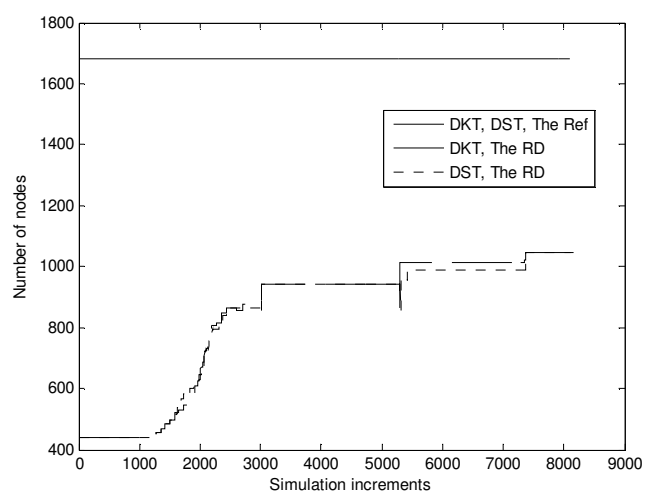

Fig. 7. The history of mesh growth during the implementation simulation for both elements.

\section{CONCLUSIONS}

The overall performance of the RD approach is good. It achieves $50 \%$ reduction of the computing time. A better mapping that includes the nonlinearity is required to reduce the over estimation of the equivalent plastic strain. The RD approach can adapt the mesh connectivity considering the tool path and the size of the blank.

\section{REFERENCES}

1. M. Bambach, G. Hirt, J. Ames, Modelling of optimization strategies in the incremental sheet metal forming process, In: Proc. NUMIFORM, Columbus, Ohio (2004).

2. A. Huerta, P. Diez and A. Rodriguez-Ferran, Adaptivity and error estimation, In: Proc. The $6^{\text {th }}$ International Conference on Numerical methods in Industrial Forming processes, Rotterdam (1998) 63-74.

3. T. Meinders, Developments in numerical simulations of real-life deep drawing process, $\mathrm{PhD}$ thesis, University of Twente, Enschede (2000).

4. J. Bonet, Error estimators and enrichment procedures for the finite element analysis of thin sheet metal forming processes, International Journal for Numerical Methods in Engineering, 34, (1994) 1573-1591.

5. R.D. Cook, D.S. Malkus, M.E. Plesha and R.J. Witt, Concepts and application of finite element analysis, $4^{\text {th }}$ edition, John Wiley \& Sons. Inc. (2002)

6. J.-L. Batoz, K.-J. Bathe and L.W. Ho, A study of threenode triangular plate bending elements, International Journal for Numerical Methods in Engineering, 18.7, (1980) 1771-1812.

7. J. L Batoz and P. Lardeur, A discrete shear triangular nine D.O.F. element for the analysis of thick to vary thin plates, International Journal for Numerical Methods in Engineering, 28.3, (1989) 533-560. 